\title{
Technology of high birefringent microstructured polymer optical fibers
}

\author{
Jan Wójcik, ${ }^{*}$ Paweł Mergo, Jacek Klimek, Grzegorz Wójcik, Krzysztof Skorupski, Janusz Pędzisz, Jarosław Kopeć \\ Department of Optical Fibres Technology, Faculty of Chemistry, Maria Curie Skłodowska University, \\ Skłodowska Sq. 3, 20-031 Lublin
}

Received March 20, 2010; accepted March 26, 2010; published March 31, 2010

\begin{abstract}
Commercially available silica glass microstructured optical fibers with birefringence caused by filling factor asymmetry may be used in optical fiber sensors. Nevertheless, their compatibility with soft polymers is rather poor. Better fitting needs to be obtained between optical fibers and materials with smaller Young modulus. High birefringent microstructured polymer optical fibers with, understandably, a relatively very small Young modulus are quite wellknown but their technology and sensor properties are rather slightly advanced [1]. The paper presents the technology of manufacturing high birefringent microstructured polymer optical fibers $\mathrm{mPOF}$ HB from polymethylmetacrylate (PMMA). The fabricated fibers were characterized from the point of optical, transmission and sensor properties.
\end{abstract}

In 1996 in our Department was elaborated the technology of side-hole HB optical fiber made from silica glass (Fig. 1a). It has been most sensitive to the pressure type of optical fibers in the last 10 years [2]. In 2008 the first photonic equivalent of a side-hole optical fiber was elaborated (Fig. 1b). This fiber has two areas with a big filling factor instead of two big holes existing in side hole fibers. This fiber was named a V-type photonic crystal fiber.

a)

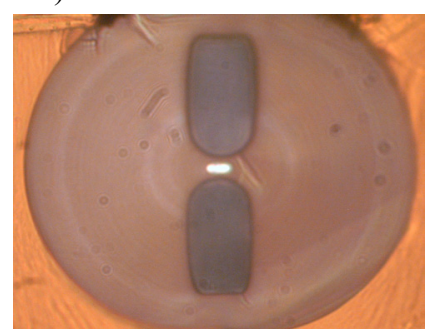

b)

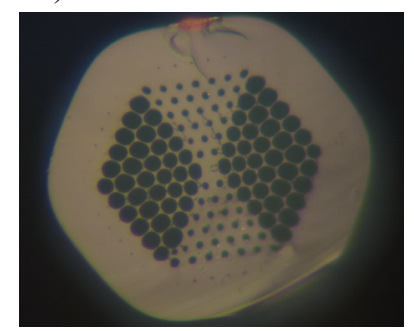

Fig. 1. Optical microscope photo of a cross section of: a) side-hole HB and b) V-type photonic crystal optical fibers. Both fibers are made from silica glass

Measurements of pressure and temperature sensitivities show its very high selectivity to pressure [3]. Nevertheless those fiber are not compatible with typical organic polymers. Therefore quite recently, in our Department, the technology of high birefringent microstructure polymer

\footnotetext{
*E-mail: wojcik@umcs.lublin.pl
}

optical fibers (mPOF HB) from polymethylmetacrylate (PMMA) was elaborated.

This technology is very similar to stack and draw processes well known from the technology of silica glass photonic crystal fibers. Our stack and draw technology of mPOF was described in [4].

Figure 2 presents the photos of cross sections of a praprefrom and perform for a fabricated $\mathrm{mPOF} H \mathrm{HB}$.

a)

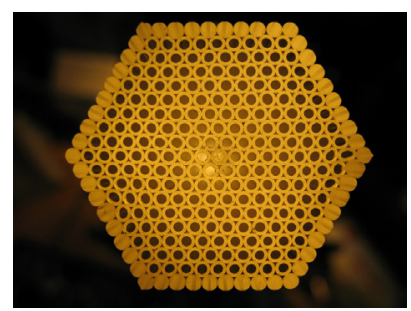

b)

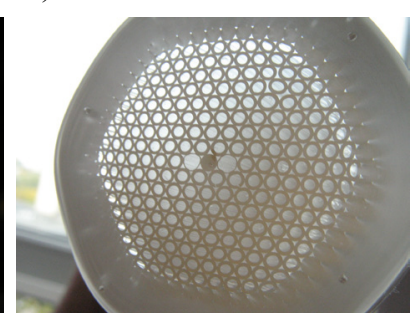

Fig. 2. Photographs of cross sections of a) praprefrom and b) perform after the overcladding process

The photonic part of a perform consists of 228 capillaries with a smaller filing factor, 2 capillaries with a bigger filling factor placed on both sides of the core and 1 rod as the core. The diameter of the prefrom after the overcladding process was equal to $40 \mathrm{~mm}$. From this preform were draw some kinds of optical fibers with a different external diameter (see Table 1). Figure 3 presents an optical microscope photo of the mPOF fiber with marked geometrical parameters.

\begin{tabular}{|c|c|c|}
\hline Fiber no. & Fiber diameter $\mathrm{P}[\mu \mathrm{m}]$ & Lattice constant $[\mu \mathrm{m}]$ \\
\hline $090729 \mathrm{P} 1$ & 500 & 19 \\
\hline $090729 \mathrm{P} 2$ & 250 & 9.5 \\
\hline $090729 \mathrm{P} 3$ & 180 & 6.8 \\
\hline $090729 \mathrm{P} 4$ & 500 & 19 \\
\hline $090729 \mathrm{P} 5$ & 250 & 9.5 \\
\hline $090729 \mathrm{P} 6$ & 180 & 6.8 \\
\hline $090729 \mathrm{P} 7$ & 150 & 5.7 \\
\hline
\end{tabular}

Table 1. Numbers of manufactured HB mPOF optical fibers, their external diameters and lattice constants. 
Spectral attenuation measured for selected fiber is relatively high (see Fig. 4b). Therefore rod without a photonic structure was used as a blank probe. Figure 4 presents spectral attenuations of fiber 090729 P5 and rod without a photonic structure.

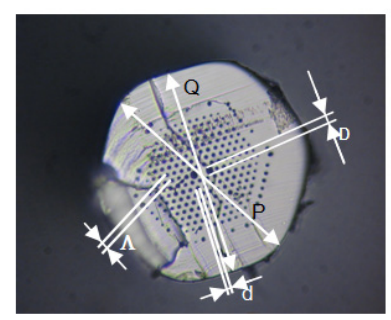
090729P7
$\mathrm{P}=157 \mu \mathrm{m}$
$\mathrm{Q}=146 \mu \mathrm{m}$
$\Lambda=5,7 \mu \mathrm{m}$
$\mathrm{D}=5 \mu \mathrm{m}$
$\mathrm{d}=2,5 \mu \mathrm{m}$
$\mathrm{d} / \Lambda=0,44$
$\mathrm{D} / \Lambda=0,88$

Fig. 3. Optical microscope photo of cross sections of mPOF HB with marked geometrical parameters: $\mathrm{P}, \mathrm{Q}-$ equivalents of fiber diameter, $\mathrm{D}$

- diameter of large holes, $\mathrm{d}$ - diameters of small holes, $\Lambda$ - lattice constant. a)

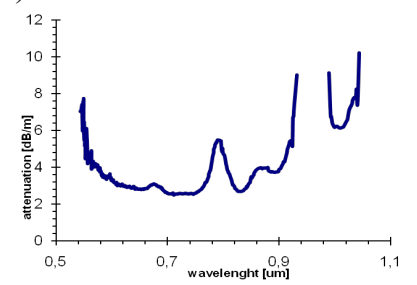

b)

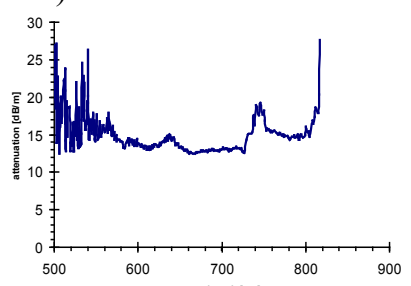

Fig. 4. Spectral attenuations of a) PMMA rod without a photonic structure - blank probe, b) mPOF fiber no. 090729 P5.

The above measurement show smaller attenuation of a rod without a photonic structure than in the case of an optical fiber. We suppose that this fact is partially caused by the presence of a photonic structure and partially by impurities introduced in all technological stages (fiber is fabricated by stack and draw method in clean rooms class 1000 only [2], technology of blank probe is much easier).

For manufactured fibers the beat lengths were measured by the lateral force method. For example, for fiber no. 090729 P7 the measured beat length was equal to $65 \mathrm{~mm}$, and the birefringence equal to $9,74 * 10^{-6}$ was calculated (wavelength $633 \mathrm{~nm}$ ). Also phase sensitivity to temperature and pressure was measured. Figure 5 presents the used measurement setup.

Phase sensitivity to temperature was measured in three different temperature ranges: $\sim 10^{\circ} \mathrm{C}-95^{\circ} \mathrm{C}, \sim 10^{\circ} \mathrm{C}-80{ }^{\circ} \mathrm{C}$ i $\sim 10^{\circ} \mathrm{C}-64{ }^{\circ} \mathrm{C}$, in the cycle hitting - cooling, for three samples of mPOF fiber no. 090729P7. Figures 6, 7 and 8 present measurement results.

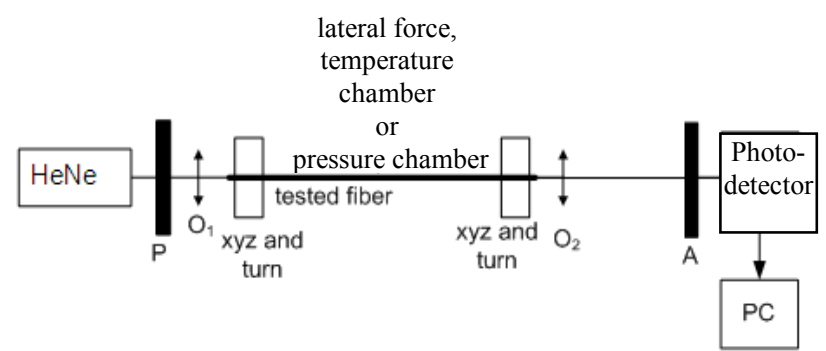

Fig. 5. Polarimetric setup for beat length, phase sensitivity for temperature and pressure measurements
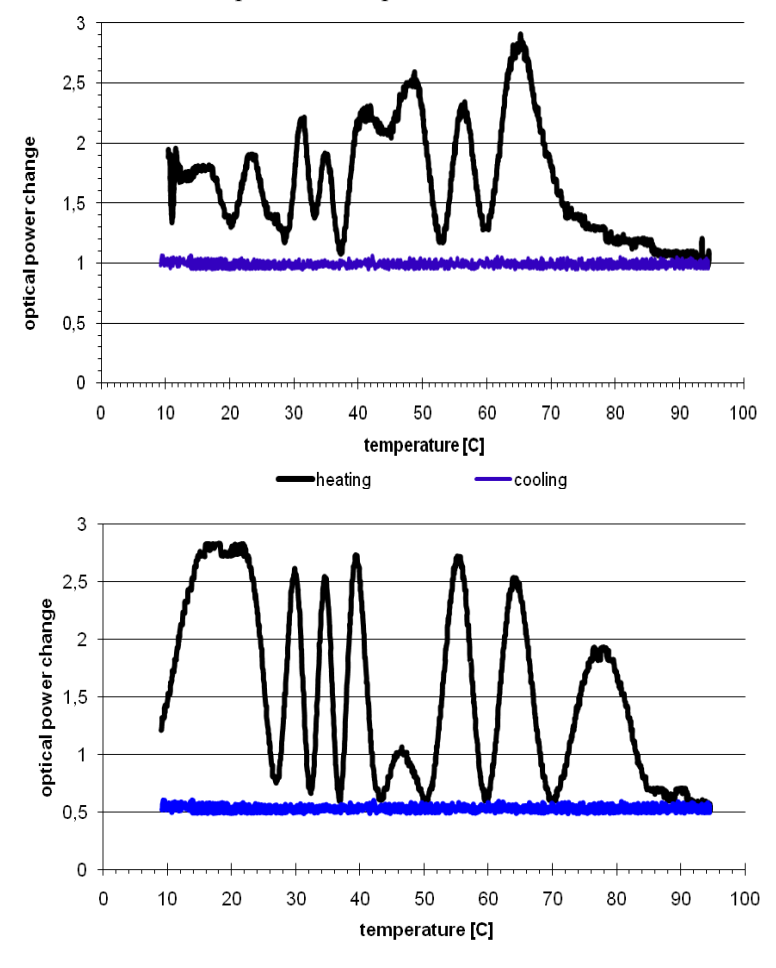

Fig. 6. Measurements made up to $95^{\circ} \mathrm{C}$, in a temperature near vitrification was observed decay of light power

The performed measurement shows that if the $\mathrm{mPOF}$ fiber was heated to a temperature near vitrification temperature $T_{g}$, the decay of light power was observed. After slow cooling (about 40min) the optical fiber didn't recover the original parameters ( no light transmission was observed). After the measurements were made, we observed the destruction of a photonic structure in a fiber cross section. 

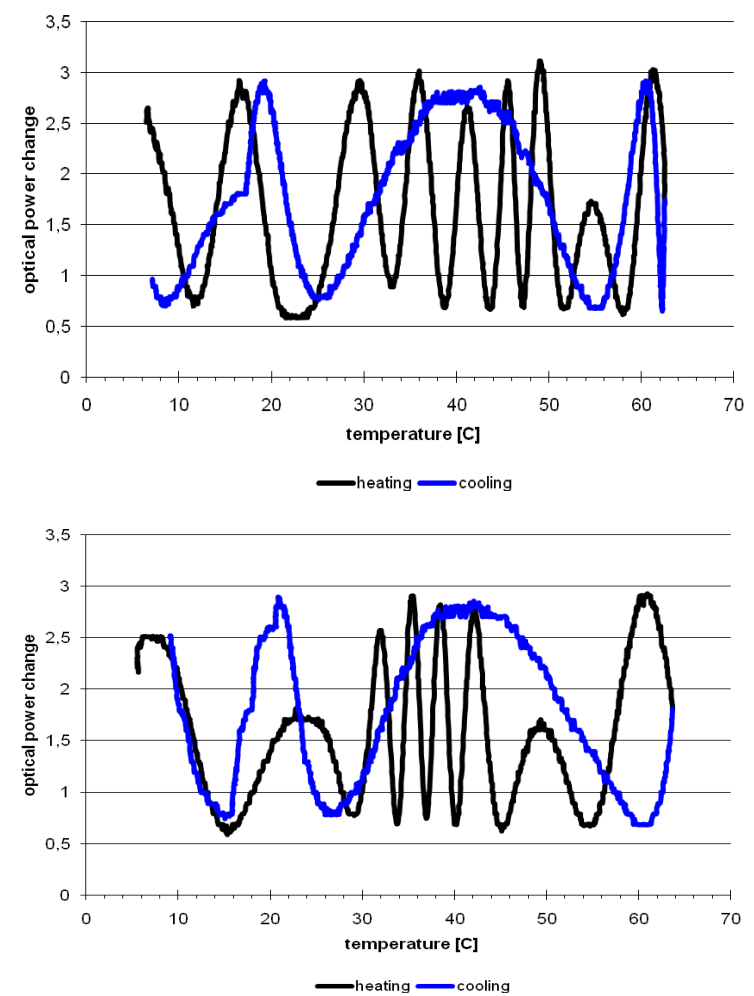

Fig. 7. Measurements made up to $64^{\circ} \mathrm{C}$, the upper graph presents the results of the first measurement, the bottom presents the results of the second measurement made right after the first one.

In measurements made up to a temperature of $80^{\circ} \mathrm{C}$ (Fig. 8) slow material relaxation was observed in the cooling process.. The result of the second measurement of the same fiber did not confirm the result of the first measurement. Probably, partial destruction of an optical fiber occurred (it did not confirm with the optical microscope measurement of geometrical properties).

In measurements made up to a temperature of $64^{\circ} \mathrm{C}$ (Fig. 7) relaxation goes faster in the cooling process than in the case of measurements up to $80^{\circ} \mathrm{C}$. The result of the second measurement of the same fiber was the same as the result of the first measurement.

Sample manufactured mPOF optical fiber was subjected to the pressure in the 0 to $6.1 \mathrm{MPa}$ range in a temperature equal to $25^{\circ} \mathrm{C}$. Measurements was repeated for the same fiber. Very good repeatability of measurements was obtained. The sensitivity to pressure for fiber no. 090729 P7 measured with interferometric method [4] is equal to $28 \mathrm{rad} /\left(\mathrm{MPa}^{*} \mathrm{~m}\right)$. It is a very high sensitivity in the context of rather a not very high beat length. We did not observe any destruction of $\mathrm{mPOF}$ fibers even a pressure of $6.1 \mathrm{MPa}$.

On the basis of V type silica glass PCF fiber (photonic equivalent of a side-hole optical fiber) two different kinds
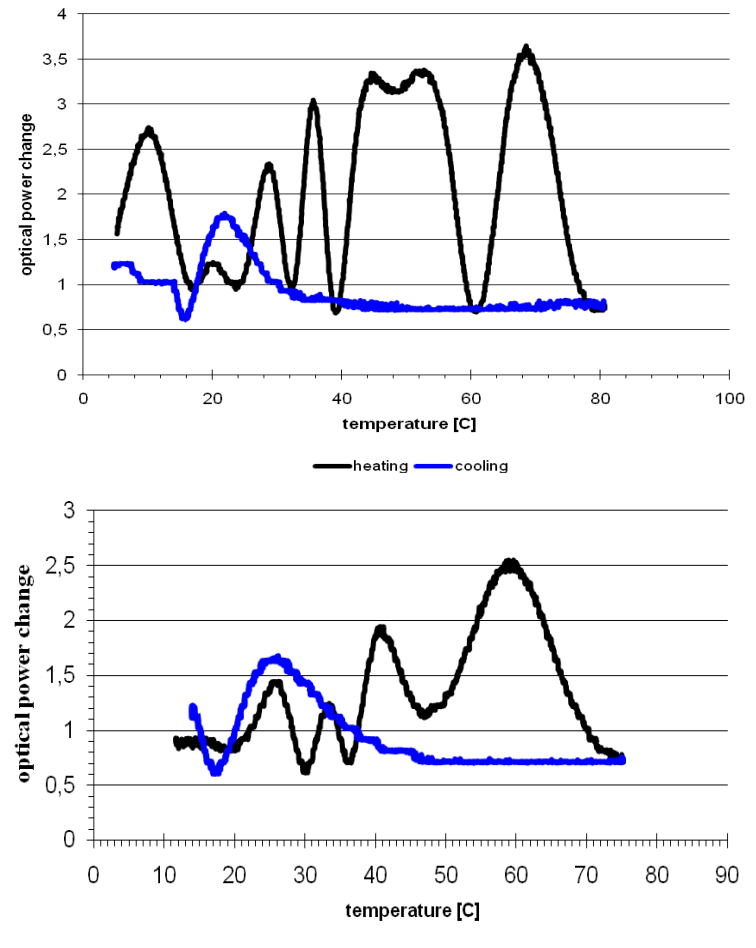

-heating -cooling

Fig. 8. Measurements made up to $80^{\circ} \mathrm{C}$, the upper graph presents the results of the first measurement, the bottom presents the results of the second measurement.

of microstructured polymer optical fibers were manufactured. One of them is named LV HB mPOF and the other is named $-\mathrm{SV}$ HB mPOF (see Fig. 9 a and c).

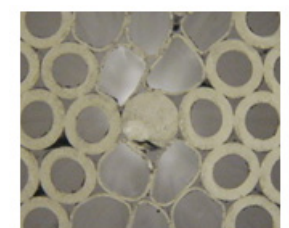

a)

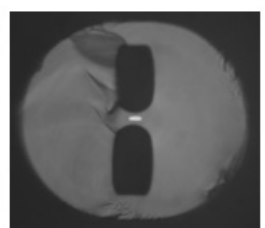

b)

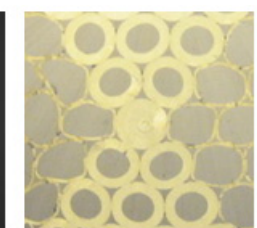

c)
Fig. 9. Optical microscope photos of cross sections of: a) perform of the LV HB mPOF, b) side-hole optical fiber, c) perform of SV HB mPOF.

Some optical fibers were manufactured. Currently, these fibers are being measured. The results will be published in the near future.

This work was financed by own investigation of Maria Curie Sklodowska University.

\section{References}

1. M. Large, C. Poladian, L. Barton, and G. Eijkelenborg, Microstructured Polymer Optical Fibers, Springer, 2008.

2. Wojcik J. at all, Proceedings SPIE 3731, 88 (1999).

3. Wojcik J., at all, Photonic Letters of Poland 2 (1), (2010).

4. Wojcik J., at all, Lightguides and Their Applications Conference TAL2009 (in press). 